\title{
MenB-FHbp Vaccine Protects Against Diverse Meningococcal Strains in Adolescents and Young Adults: Post Hoc Analysis of Two Phase 3 Studies
}

\author{
Johannes Beeslaar · Judith Absalon · Annaliesa S. Anderson • \\ Joseph J. Eiden · Paul Balmer • Shannon L. Harris - Thomas R. Jones • \\ Robert E. O'Neill · Jean-Louis Pregaldien · David Radley • \\ Roger Maansson · John Ginis · Amit Srivastava · John L. Perez
}

Received: October 30, 2019 / Published online: July 22, 2020

(C) Pfizer Inc. 2020

\section{ABSTRACT}

Introduction: Two phase 3 studies in adolescents and young adults demonstrated that MenB-FHbp, a meningococcal serogroup B (MenB) vaccine, elicits protective immune responses after 2 or 3 doses based on serum bactericidal antibody assays using human complement (hSBA) against 4 primary and 10 additional diverse, vaccine-heterologous MenB test strains. Lower limits of quantitation (LLOQs; titers $1: 8$ or $1: 16$; titers $\geq 1: 4$ correlate with protection) were used to evaluate

Digital Features To view digital features for this article go to https://doi.org/10.6084/m9.figshare.12410216.

Electronic supplementary material The online version of this article (https://doi.org/10.1007/s40121020-00319-0) contains supplementary material, which is available to authorized users.

J. Beeslaar $(\square)$

Vaccine Clinical Research and Development, Pfizer Ltd UK, Hurley, UK

e-mail: Johannes.Beeslaar@pfizer.com

J. Absalon · J. J. Eiden · D. Radley

Vaccine Clinical Research and Development, Pfizer Inc, Pearl River, NY, USA

A. S. Anderson · S. L. Harris · T. R. Jones ·

R. E. O'Neill

Vaccine Research and Development,

Pfizer Inc, Pearl River, NY, USA responses to individual strains and all 4 primary strains combined (composite response). A post hoc analysis evaluated percentages of subjects with protective responses to as many as 8 strains combined (4 primary plus additional strains). Methods: Immune responses were measured using hSBAs against 4 primary strains in adolescents ( $n=1509$, MenB-FHbp; $n=898$, hepatitis A virus vaccine/saline) and young adults ( $n=2480$, MenB-FHbp; $n=824$, saline) receiving MenB-FHbp or control at 0, 2, and 6 months. Ten additional strains were evaluated in subsets of subjects from approximately 1800

P. Balmer · A. Srivastava

Vaccine Medical Development, Scientific and Clinical Affairs, Pfizer Inc, Collegeville, PA, USA

\section{J.-L. Pregaldien}

Vaccine Clinical Research and Development, Pfizer Inc, Brussels, Belgium

R. Maansson · J. L. Perez

Vaccine Clinical Research and Development, Pfizer Inc, Collegeville, PA, USA

J. Ginis

Vaccine Research and Development,

Pfizer Inc, Collegeville, PA, USA 
MenB-FHbp recipients across both studies. Percentages of subjects with hSBA titers $\geq$ LLOQ for different numbers of primary strains or primary plus additional strains combined (7 or 8 strains total per subset) were determined before vaccination, 1 month post-dose 2 , and 1 month post-dose 3 .

Results: Across the panel of primary plus additional strains, at 1 month post-dose 3 , titers $\geq$ LLOQ were elicited in $93.7-95.7 \%$ of adolescents and 91.7-95.0\% of young adults for $\geq 5$ test strains combined and in $70.5-85.8 \%$ of adolescents and $67.5-81.4 \%$ of young adults for $\geq 7$ strains combined. Among adolescents, $99.8 \%, 99.0 \%, 92.8 \%$, and $82.7 \%$ had titers $\geq$ LLOQ against at least 1, 2, 3, and all 4 primary strains, respectively; corresponding percentages for young adults were 99.7\%, 97.7\%, 94.0\%, and $84.5 \%$.

Conclusions: Results support the ability of MenB-FHbp to provide broad coverage against MenB strains expressing diverse FHbp variants.

Trial Registration: ClinicalTrials.gov identifiers NCT01830855, NCT01352845.

Keywords: Adolescents; Bactericidal activity; Broad coverage; FHbp; hSBA; MenB-FHbp vaccine; Meningococcal disease; Meningococcal serogroup B; Neisseria meningitidis; Young adults

\section{Key Summary Points}

\section{Why carry out this study?}

In many regions of the world, meningococcal serogroup B (MenB) is the predominant cause of invasive meningococcal disease.

MenB disease-causing strains are characterized by the diversity of antigens expressed, complicating the development and evaluation of MenB vaccines.

It is essential to provide evidence that MenB vaccines protect against the diversity of MenB disease-causing strains.

This post hoc analysis evaluates the extent of MenB-FHbp vaccine coverage across diverse MenB strains in adolescent and young adults using data from 2 phase 3 studies.

\section{What was learned from the study?}

One month after completion of a 3-dose series of MenB-FHbp, a high percentage of subjects showed protective responses against at least 5 (91.7-95.7\%) and 7 (67.5-85.8\%) of the test strains combined.

These results highlight the broad protection elicited by MenB-FHbp against a diverse array of MenB strains.

\section{INTRODUCTION}

Invasive meningococcal disease (IMD) is an infection caused by the bacterium Neisseria meningitidis [1]. Although IMD incidence is generally low ( 0.1 per 100,000 population in the United States and 0.6 per 100,000 population in the European Union/European Economic Area in 2018 [2, 3]), disease progression can be rapid and the consequences can be severe $[4,5]$. The case fatality rate of IMD ranges from 8 to $23 \%$, and nearly $20 \%$ of survivors suffer long-term 
sequelae such as limb loss or hearing impairment $[1,5,6]$. The rapid progression of IMD coupled with its potentially devastating consequences underscore the importance of preventing disease by vaccination.

The epidemiology of IMD is dynamic, varying temporally, geographically, and by agebased population, thereby necessitating the adaptation of vaccination strategies for disease prevention. For instance, although nearly all IMD is caused by meningococcal serogroups $A$, $\mathrm{B}, \mathrm{C}, \mathrm{W}, \mathrm{X}$, and $\mathrm{Y}$ [7], meningococcal serogroup $B$ (MenB) is currently dominant in diverse regions, such as North America, the European Union, Japan, Israel, and Australia $[2,8,9]$.

Currently available meningococcal vaccines include capsular polysaccharide conjugate vaccines targeting serogroups $\mathrm{A}, \mathrm{C}, \mathrm{W}$, and $\mathrm{Y}$ (MenACWY vaccines) [10-12] and, more recently, MenB vaccines $[13,14]$. Because the MenB capsular polysaccharide is poorly immunogenic $[15,16]$, licensed MenB vaccines [13, 14] required identification of surface-exposed protein antigens to elicit broadly protective bactericidal antibodies, which correlate with protection against IMD $[17,18]$, against diverse disease strains. One such vaccine antigen is factor $\mathrm{H}$ binding protein (FHbp). Importantly, $>90 \%$ of MenB strains worldwide express sufficient levels of FHbp to induce vaccine-elicited complement-mediated bactericidal activity [19].

With the use of protein antigens for the development of contemporary MenB vaccines, the need arose to demonstrate breadth of coverage against diverse circulating MenB diseasecausing strains. Unlike capsular polysaccharides that have limited variability across strains, protein antigens can have multiple variants and exhibit differential expression [20, 21]. For FHbp in particular, sequences can be highly variable [22]. However, it is not feasible to evaluate breadth of coverage against strains expressing all the known variants ( $>1000$ peptide alleles for FHbp) [23]. Therefore, a rational approach is to evaluate MenB strain panels that are representative of the global diversity of MenB disease-causing strains to provide confidence that the vaccine will elicit protective coverage.

MenB-FHbp (Trumenba ${ }^{\circledR}$, bivalent rLP2086; Pfizer Inc, Philadelphia, PA, USA) is a MenB vaccine composed of 2 recombinant lipidated $\mathrm{FHb}$ proteins, one from each subfamily (variant A05 from subfamily A and variant B01 from subfamily B), and was initially approved in the United States in October 2014 for the prevention of IMD in individuals 10-25 years of age $[13,24]$. More recently, MenB-FHbp was approved in Europe for individuals $\geq 10$ years of age [25], and has also been approved in a number of other countries [26-28].

The clinical development of MenB-FHbp relied on a diverse panel of MenB test strains to confirm broadly protective immune responses. These included 4 primary test strains, 2 each from FHbp subfamily A and subfamily B, representative of the epidemiologic diversity of disease-causing MenB isolates and expressing FHbps that differ from vaccine antigens $[29,30]$. Ten additional test strains were selected from a pool collected in collaboration with national reference laboratories to supplement data on vaccine-elicited protective immune responses obtained with the primary strains, and to examine whether immune responses to the primary strains were predictive of responses against other antigenically diverse MenB test strains expressing prevalent FHbp variants [29]. Collectively, these 14 test strains contain FHbp sequences expressed by approximately $80 \%$ of circulating disease strains in the United States and Europe. Importantly, for both diverse strain panels, immune responses in serum bactericidal antibody assays using human complement (hSBA) were assessed in which titers $1: 8$ or $1: 16$ (depending on the test strain) were used as the lower limit of quantitation (LLOQ) [29]. These titers represent a more conservative threshold than the recognized correlate of protection for meningococcal disease (i.e., an hSBA titer of $\geq 1: 4)[17,18]$. Additionally, the percentages of subjects with titers $\geq$ LLOQ against the 4 primary strains combined were evaluated [29]; this measure, termed the composite response, provides a stringent representation of breadth of coverage of MenB-FHbp.

Two pivotal phase 3 studies, one in adolescents and the other in young adults, provided key immunogenicity and safety data for MenBFHbp [29]. In both studies, MenB-FHbp recipients were vaccinated at 0,2 , and 6 months. 
Immune responses were measured by hSBAs against the 4 primary (assessed in all subjects) and 10 additional (assessed in random subsets of subjects) MenB test strains. Using the 1:8 and 1:16 LLOQ thresholds, results for the modified intent-to-treat populations showed that 1 month after the third MenB-FHbp dose, $86.4-99.5 \%$ and $75.3-98.7 \%$ of adolescents, depending on strain, had hSBA titers $\geq$ LLOQ against each of the 4 primary test strains and each of the 10 additional test strains, respectively; $82.7 \%$ had hSBA titers $\geq$ LLOQ against all 4 primary test strains combined (composite response). One month post-dose 2 , percentages were $64.0-99.0 \%$ (primary strains), 58.8-99.0\% (additional strains), and 53.7\% (composite response). For young adults, percentages at 1 month post-dose 3 were $87.1-99.3 \%$ (primary strains), 71.5-99.3\% (additional strains), and $84.5 \%$ (composite response); at 1 month postdose 2 , they were $67.3-97.4 \%$ (primary strains), $51.5-98.0 \%$ (additional strains), and 63.3\% (composite response). Notably, immune responses to the 4 primary test strains predicted responses to the 10 additional strains in the 2 pivotal phase 3 studies.

The high rates of vaccine-elicited immune responses to diverse MenB strains using LLOQ thresholds more stringent than the recognized correlate of protection for meningococcal disease, coupled with high rates of composite responses [29], support the breadth of protection provided by MenB-FHbp. Additionally, most individuals without demonstrable hSBA titers $\geq$ LLOQ for a particular strain still mounted protective responses to other test strains.

To further quantify these results, this post hoc analysis of data from the 2 pivotal phase 3 studies evaluated the percentages of subjects with hSBA titers $\geq$ LLOQ against as many as 8 strains (4 primary plus additional test strains) combined. In addition, composite responses to at least 1,2,3, and all 4 primary test strains were evaluated. Ultimately, this analysis sought to further substantiate the breadth of response against diverse MenB strains in MenB-FHbp recipients and examined whether there were any vaccinees who did not respond to any test strains.

\section{METHODS}

\section{Populations for Analysis}

In the adolescent lot consistency study (NCT01830855), subjects 10-18 years of age were randomized in a 5:2:2:3 ratio to receive 1 of 3 lots of MenB-FHbp or hepatitis A virus vaccine (HAV)/saline at 0,2 , and 6 months [29]. The current analysis reports results only from the MenB-FHbp group that received the first lot of MenB-FHbp and for which all primary test strains were evaluated ( $n=1509$ randomized), as well as the HAV/saline group ( $n=898$ randomized); subjects receiving lots 2 and 3 were not included because their sera were tested against only 2 of the primary test strains to meet a lot consistency objective. In the young adult study (NCT01352845), subjects 18-25 years of age were randomized in a $3: 1$ ratio to receive MenB-FHbp or saline at 0,2 , and 6 months; because all primary test strains were evaluated in all subjects, results from both groups ( $n=2480$ and $n=824$ randomized, respectively) are included in the current analysis [29]. Protocols for both previously conducted studies used for analysis here were approved by the institutional review boards and/or independent ethics committees for the respective investigational centers that participated. Written informed consent was obtained from all subjects or their legal guardian prior to enrollment and any study-related procedures. The present analysis is based on existing data collected from these studies and therefore did not involve any new data collection or new studies with human subjects performed by any of the authors.

Consistent with the primary study publication [29], the modified intent-to-treat populations were used for the current analyses. This included all subjects who were randomized and had at least 1 valid and determinate assay result related to the analysis for each study. To support the current analysis, all analyses were repeated in the per-protocol population (i.e., evaluable immunogenicity population), which included all randomized subjects who were eligible for the study, received the correct study product, had post-vaccination blood 
draws within the pre-specified intervals, had valid and determinate assay results, and had no major protocol violations. Of note, immunogenicity findings in the primary study publication were consistent between the modified intent-to-treat and per-protocol populations [29].

\section{Immunogenicity Evaluations}

Immune responses were measured using hSBA assays, the accepted surrogate for MenB vaccine efficacy [31]. The 4 primary test strains expressed vaccine-heterologous $\mathrm{FHbp}$ variants A22, A56, B24, and B44; the 10 additional test strains expressed vaccine-heterologous FHbp variants A06, A07, A12, A15, A19, A29, B03, $\mathrm{B} 09, \mathrm{~B} 15$, and B16. The LLOQs were determined as titers 1:16 for the strains expressing FHbp variants A22, A06, A12, and A19 and 1:8 for the strains expressing FHbp variants A56, B24, B44, A07, A15, A29, B03, B09, B15, and B16.

All subjects from both studies were tested for immune responses against the 4 primary test strains. Approximately 900 MenB-FHbp recipients from each study (approximately 1800 subjects total) were also tested for bactericidal responses against additional strains. Specifically, these 900 subjects were divided into 3 subsets of 300 subjects each within each study, with each subset tested against a unique group of strains, including the 4 primary strains plus 3 or 4 of the additional test strains; thus, subjects in all subsets were tested against either 7 (2 subsets) or 8 strains (1 subset) in total. The specific additional strains included for testing in each subset are listed in Table S1 in the Electronic Supplementary Material (ESM). Immune sera from subjects in each subset were tested in individual hSBAs specific to the strains assigned to that subset before vaccination and at 1 month post-dose 3 (i.e., following the full 0,2 , and 6 month schedule). Fewer subjects in each subset had sera tested at 1 month post-dose 2 (i.e., after receiving vaccinations at 0 and 2 months) due to limited supply of assay reagents. The percentages of subjects responding to different numbers of strains were then evaluated for each subset. The percentages of subjects with hSBA titers $\geq$ LLOQ for at least 1 ,
2, 3, and all 4 primary test strains were also evaluated at various time points for each study. For analyses of primary and primary plus additional test strains, strains were not considered in a particular order; therefore, the specific strains for which an individual had titers $\geq$ LLOQ for at least $1,2,3,4,5,6,7$, or 8 strains likely varied between individuals in each responder group (e.g., while multiple individuals responded to at least 5 strains, the 5 strains with titers $\geq$ LLOQ were not necessarily identical across these individuals).

For both the primary and additional strains, only subjects with valid and determinate assay results for all strains tested in a given subset were included in the corresponding analysis. Results are reported as percentages of subjects with hSBA titers $\geq$ LLOQ or $\geq 1: 4$ for different numbers of test strains combined along with 95\% CIs using the Clopper-Pearson method.

\section{RESULTS}

\section{Responders to Primary Plus Additional Test Strains}

Among adolescents evaluated for immune responses against the primary strains and 3 or 4 additional strains, at 1 month post-dose 3 (i.e., 1 month after receiving MenB-FHbp doses at 0,2 , and 6 months), 93.7-95.7\% of subjects (depending on subset) had titers $\geq$ LLOQ for at least 5 strains combined; percentages were $70.5-85.8 \%$ for at least 7 strains combined and $71.9 \%$ for 8 strains combined (Fig. 1). At 1 month post-dose 2 (i.e., 1 month after receiving MenB-FHbp doses at 0 and 2 months), $74.7-81.2 \%$ of subjects had titers $\geq$ LLOQ for at least 5 strains combined, $44.3-57.0 \%$ had titers $\geq$ LLOQ for at least 7 strains combined, and $45.6 \%$ had titers $\geq$ LLOQ for 8 strains combined. Few subjects had pre-vaccination hSBA titers $\geq$ LLOQ for at least $5(3.0-5.8 \%)$, at least $7(0.4-1.7 \%)$, and $8(0.8 \%)$ test strains combined.

Among young adults, post-vaccination percentages of subjects with titers $\geq$ LLOQ for at least 5 and at least 7 strains combined were similar to those for adolescents. At 1 month 
a Subset 1

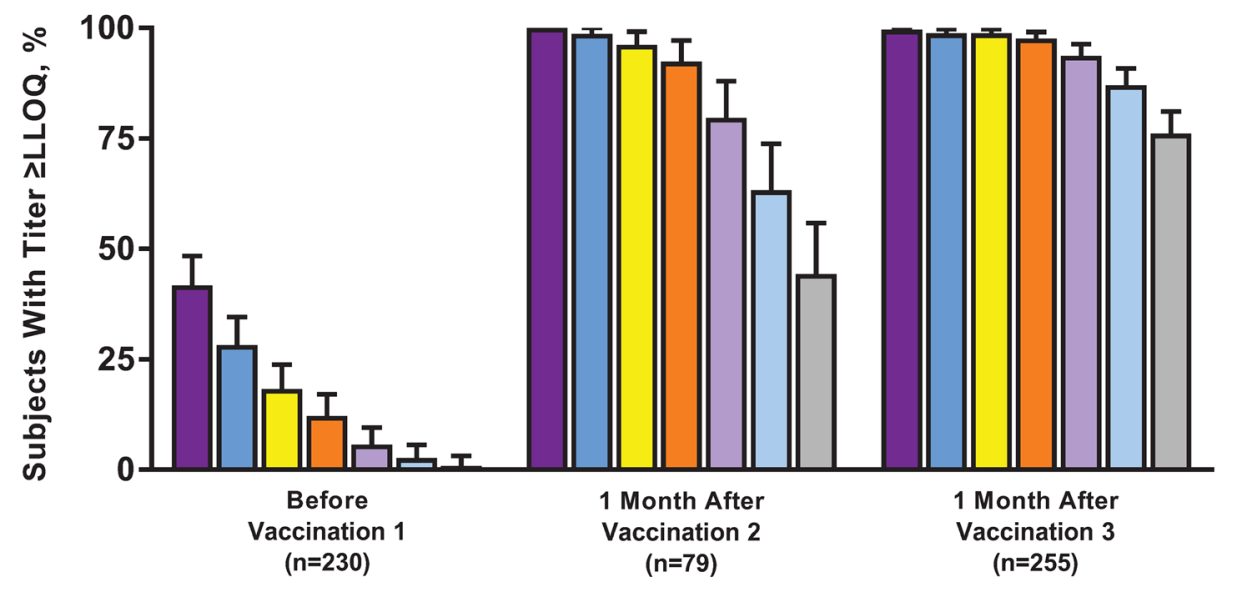

\section{b Subset 2}

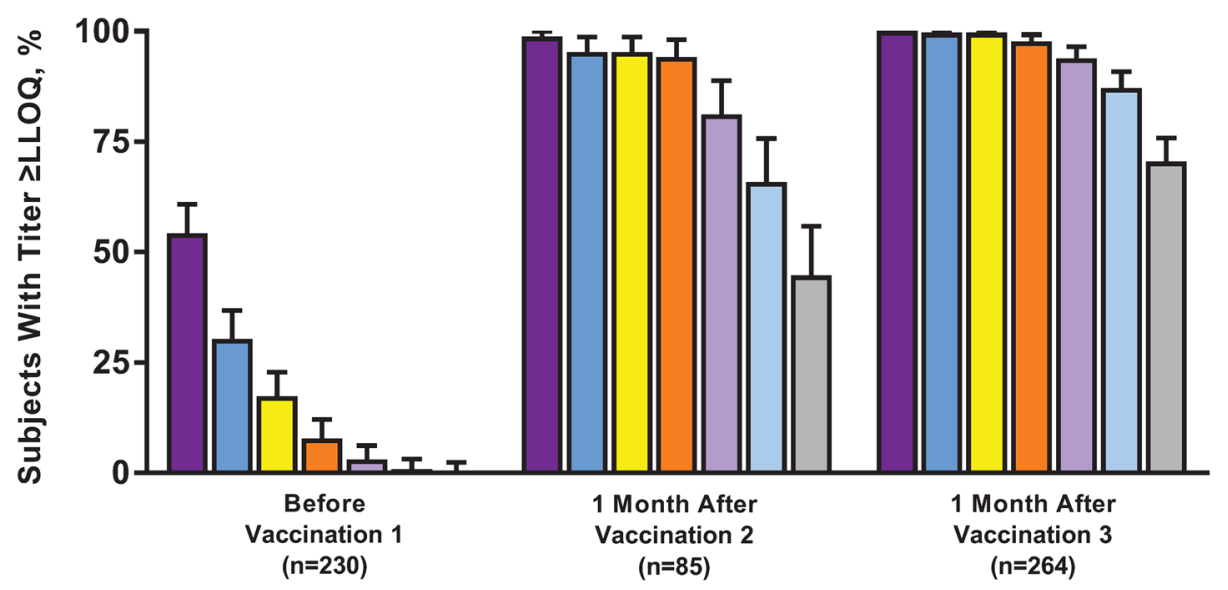

\section{c Subset 3}

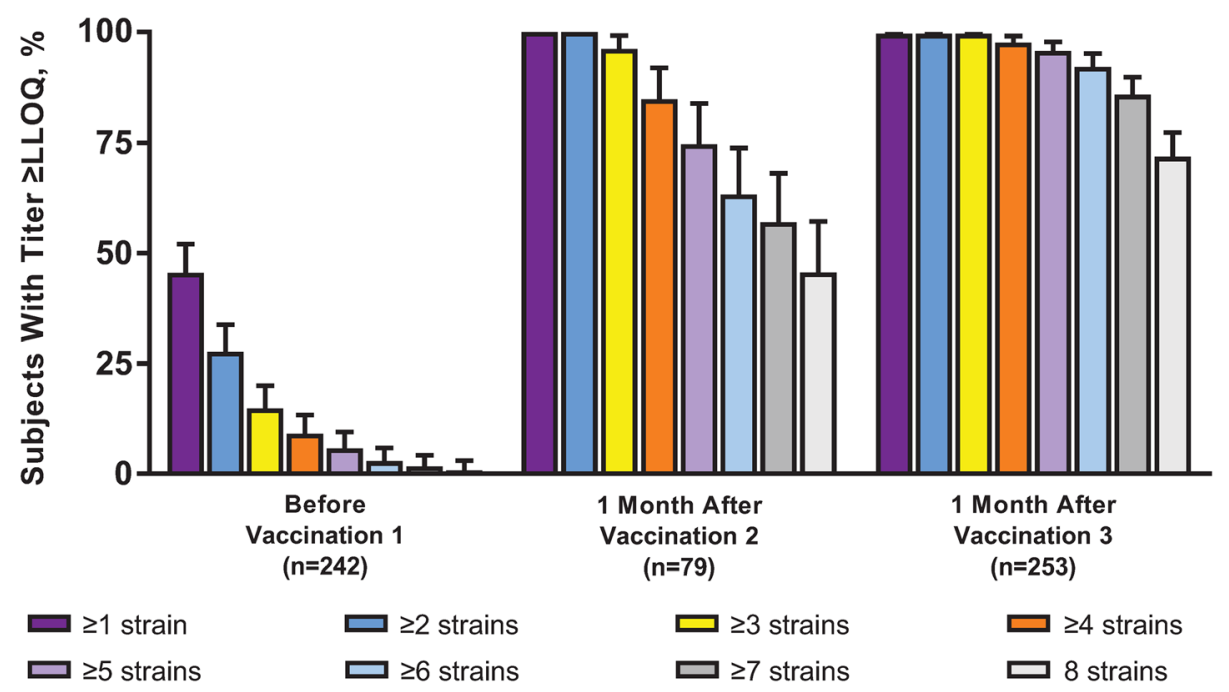


4Fig. 1 Percentage of adolescent responders with hSBA titers $\geq$ LLOQ to different numbers of primary and additional MenB test strains before MenB-FHbp vaccination, 1 month after 2 doses ( 0,2 months), and 1 month after 3 doses (0, 2, 6 months). a Subset 1; b subset 2; c subset 3. Evaluation included up to 8 test strains. Subset 1 included the primary test strains (expressing FHbp variants A22, A56, B24, and B44) and additional test strains expressing FHbp variants A15, A19, and A29. Subset 2 included the primary test strains and additional test strains expressing FHbp variants A06, A07, and A12. Subset 3 included the primary test strains and additional test strains expressing FHbp variants B03, B09, B15, and B16. The number of subjects included at each time point is indicated below the $x$-axis. Fewer subjects were evaluated post-dose 2 than at other time points because of limited supply of qualified assay reagents. $\mathrm{FH} b \mathrm{p}$ factor $\mathrm{H}$ binding protein, $h S B A$ serum bactericidal antibody assay using human complement, $L L O Q$ lower limit of quantitation, MenB Neisseria meningitidis serogroup B. Error bars represent the 95\% CIs calculated using the ClopperPearson method

post-dose 3, 91.7-95.0\% of subjects (depending on subset) had titers $\geq$ LLOQ for at least 5 strains combined; percentages were $67.5-81.4 \%$ for at least 7 and $66.2 \%$ for 8 strains combined (Fig. 2). At 1 month post-dose 2, 74.2-87.2\% of subjects had titers $\geq$ LLOQ for at least 5 strains combined, $47.1-61.7 \%$ had titers $\geq$ LLOQ for at least 7 , and $46.1 \%$ had titers $\geq$ LLOQ for 8 strains combined. Pre-vaccination titers $\geq$ LLOQ against various numbers of primary and additional test strains among young adults were $11.0-21.3 \%$ for at least $5,0.8-9.1 \%$ for at least 7 , and $5.3 \%$ for 8 strains combined.

Percentages of responders among the subset of subjects with pre-vaccination titers $<$ LLOQ for all primary plus additional strains are presented in the Supplemental Results in the ESM. When applying the recognized correlate of protection for meningococcal disease (i.e., hSBA titer $\geq 1: 4$ ), percentages of responders were slightly higher at post-vaccination time points compared with analyses using hSBA titers $\geq$ LLOQ (see Table S2 in the ESM).

\section{Responders to Primary Test Strains}

At 1 month post-dose 3, percentages of adolescent MenB-FHbp recipients with hSBA titers $\geq$ LLOQ for at least $1,2,3$, and all 4 primary test strains combined were $99.8 \%, 99.0 \%, 92.8 \%$, and $82.7 \%$, respectively (Fig. 3). At 1 month post-dose 2, percentages were $99.5 \%, 95.8 \%, 75.5 \%$, and $53.7 \%$, respectively. Before vaccination, only $1.4 \%$ of adolescent MenB-FHbp recipients had hSBA titers $\geq$ LLOQ for all 4 primary test strains; $42.3 \%$, $18.3 \%$, and $5.9 \%$ had hSBA titers $\geq$ LLOQ for at least 1,2 , and 3 test strains, respectively. Postvaccination and pre-vaccination titers were similar among adolescents receiving HAV/saline.

Among young adults, percentages of MenBFHbp recipients with hSBA titers $\geq$ LLOQ for at least $1,2,3$, and all 4 primary test strains combined were $99.7 \%, 97.7 \%, 94.0 \%$, and $84.5 \%$, respectively, at 1 month post-dose 3 (Fig. 4). At 1 month post-dose 2 , percentages were $98.5 \%$, $92.8 \%, 83.5 \%$, and $63.3 \%$, respectively. A higher percentage of young adults had pre-vaccination hSBA titers $\geq$ LLOQ to the primary test strains $(47.3 \%$ to at least 1 strain, $30.4 \%$ to at least 2 strains, $18.7 \%$ to at least 3 strains, and $6.7 \%$ to 4 strains) compared with adolescents. Similar to observations in adolescents, percentages in young adults receiving saline remained similar to those before vaccination.

Percentages of responders specifically among the subset of patients with pre-vaccination titers $<$ LLOQ for all primary strains are presented in the Supplemental Results in the ESM. Applying the correlate of protection threshold (i.e., hSBA titer $\geq 1: 4$ ) rather than the LLOQ of $1: 8$ or $1: 16$ resulted in slightly higher percentages of responders compared with analyses using hSBA titers $\geq$ LLOQ (See Table S3 in the ESM).

\section{Characteristics of Subjects Who Did Not Mount an hSBA Response to Any of the Strains Tested (Primary Plus Additional Strains)}

Very few subjects did not mount an hSBA response to any of the strains tested. Nine subjects ( 3 adolescents and 6 young adults) did not achieve hSBA titers $\geq$ LLOQ to any of the 
a Subset 1

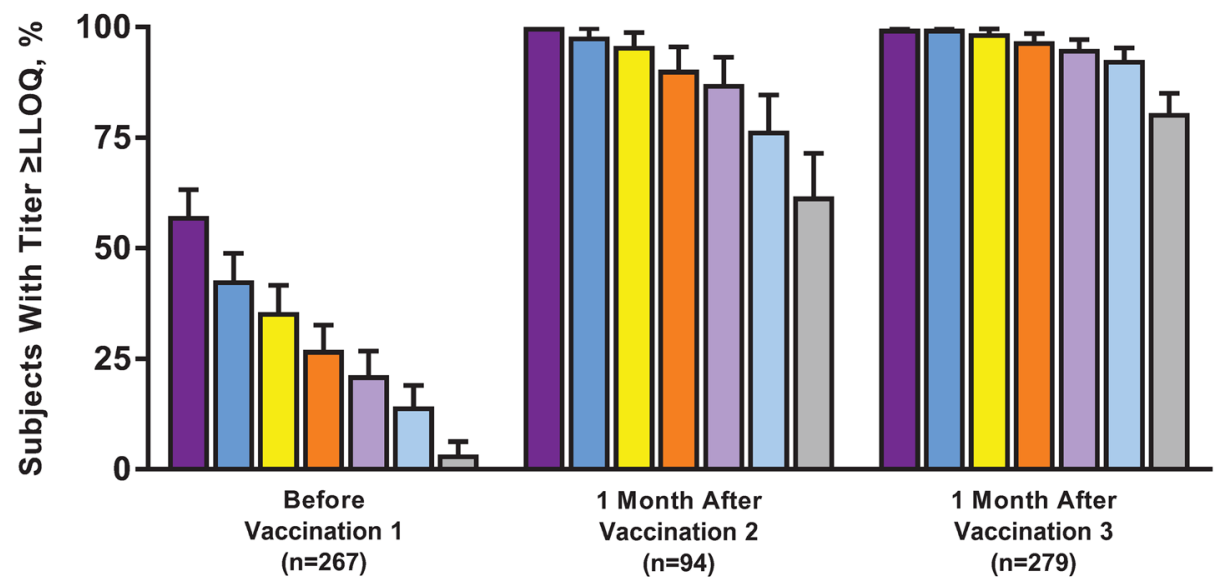

\section{b Subset 2}

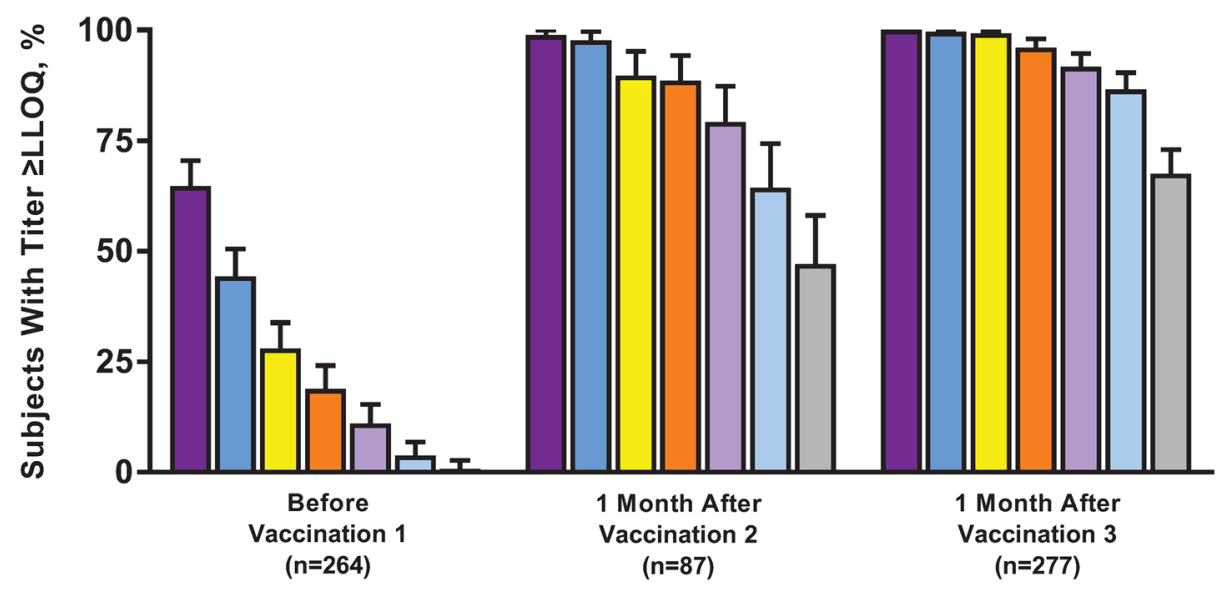

\section{c Subset 3}

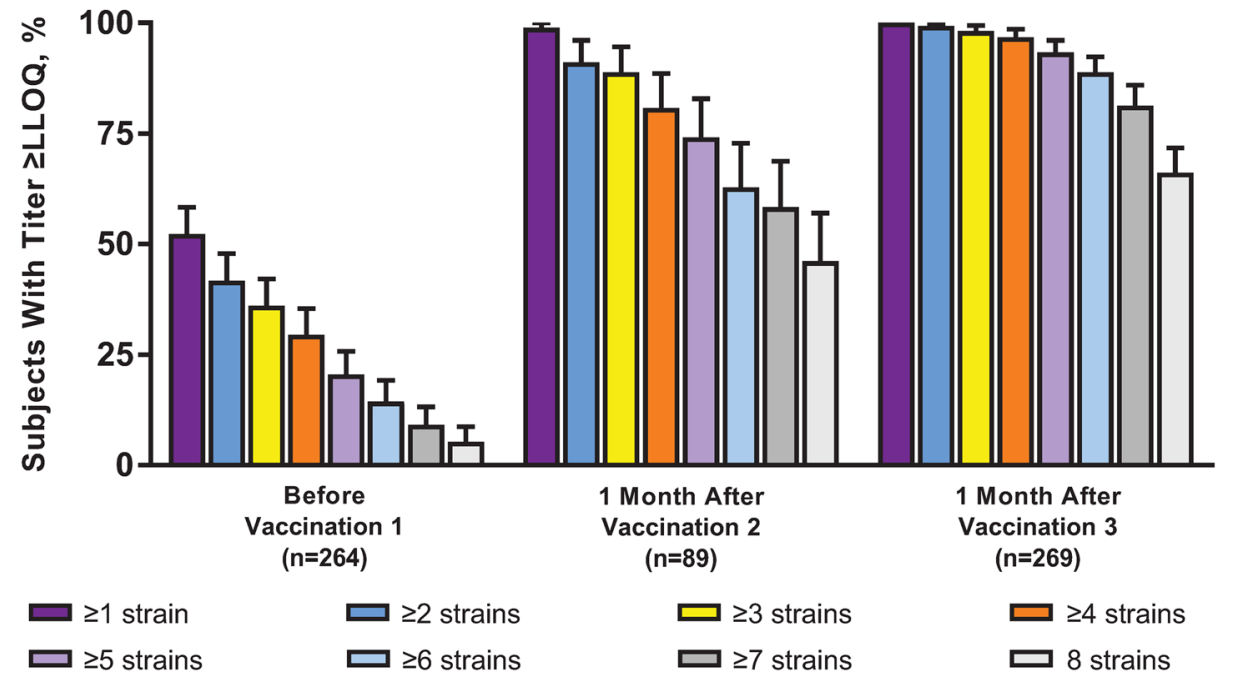


4Fig. 2 Percentage of young adult responders with hSBA titers $\geq$ LLOQ to different numbers of primary and additional MenB test strains before MenB-FHbp vaccination, 1 month after 2 doses ( 0,2 months), and 1 month after 3 doses (0, 2, 6 months). a Subset 1; b subset 2; c subset 3. Evaluation included up to 8 test strains. Subset 1 included the primary test strains (expressing FHbp variants A22, A56, B24, and B44) and additional test strains expressing FHbp variants A15, A19, and A29. Subset 2 included the primary test strains and additional test strains expressing FHbp variants A06, A07, and A12. Subset 3 included the primary test strains and additional test strains expressing FHbp variants B03, B09, B15, and B16. The number of subjects included at each time point is indicated below the $x$-axis. Fewer subjects were evaluated post-dose 2 than at other time points because of limited supply of qualified assay reagents. $F H b p$ factor $\mathrm{H}$ binding protein, $h S B A$ serum bactericidal antibody assay using human complement, $L L O Q$ lower limit of quantitation, MenB Neisseria meningitidis serogroup B. Error bars represent the 95\% CIs calculated using the ClopperPearson method primary strains tested at 1 month post-dose 3 (Table 1). Of these, 3 subjects ( 2 adolescents and 1 young adult, all female and from North America) were included in the primary/additional strain subsets and did not achieve hSBA titers $\geq$ LLOQ to any of the strains tested at 1 month post-dose 3 (Table 1: adolescent subjects $B$ and $C$; young adult subject I). The subjects with titers $<$ LLOQ for all primary test strains or all primary and additional test strains had no common medical conditions or other characteristics that might have contributed to a lack of a detectable immune response following vaccination with MenB-FHbp. None of these subjects reported prior or concomitant use of immunosuppressive drugs.

\section{DISCUSSION}

MenB vaccines must demonstrate protection against diverse pathogenic MenB strains to be clinically effective. The relatively low incidence of IMD makes it impractical to conduct a

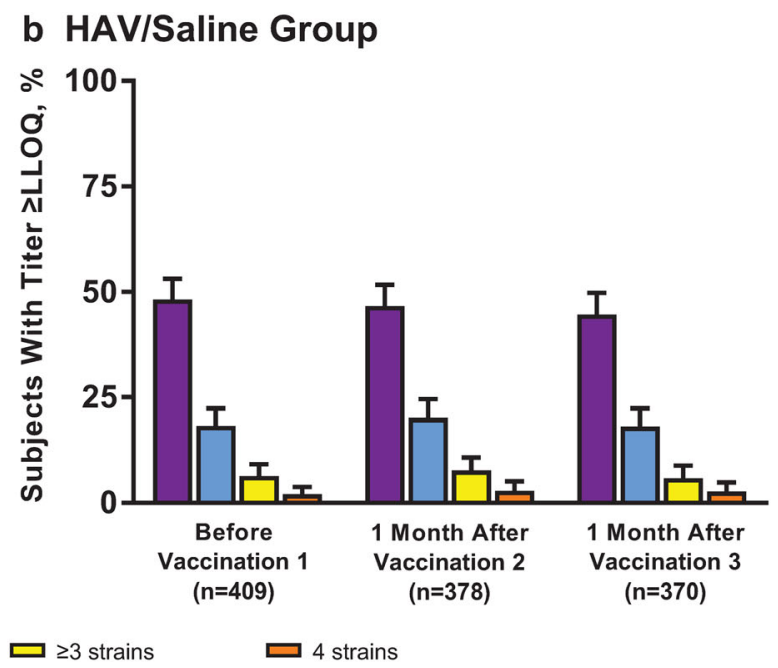

subjects included at each time point is indicated below the $x$-axis. $F H b p$ factor $\mathrm{H}$ binding protein, $H A V$ hepatitis A virus vaccine; $\quad S S B A$ serum bactericidal antibody assay using human complement, $L L O Q$ lower limit of quantitation, MenB Neisseria meningitidis serogroup B, MenB-FHbp bivalent rLP2086, Trumenba ${ }^{\circledR}$. Error bars represent the 95\% CIs calculated using the Clopper-Pearson method
Fig. 3 Percentage of adolescents in the a MenB-FHbp and $\mathbf{b} \mathrm{HAV} /$ saline groups with hSBA titers $\geq$ LLOQ to different numbers of primary MenB test strains before vaccination, 1 month after 2 doses ( 0,2 months), and 1 month after 3 doses (0, 2, 6 months). LLOQ was defined as 1:8 for the test strain expressing FHbp variant A22 and 1:16 for the test strains expressing FHbp variants A56, B24, and B44. The number of 


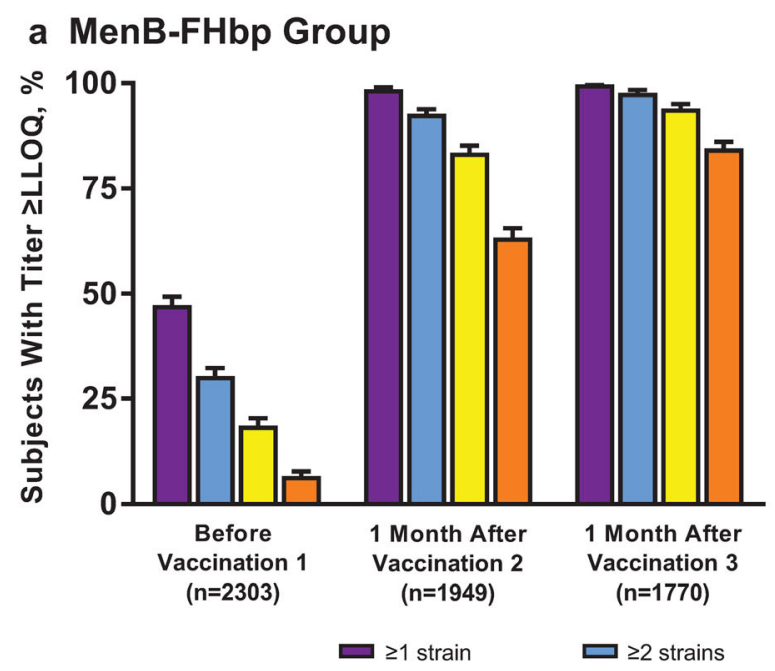

Fig. 4 Percentage of young adults in the a MenB-FHbp and b saline groups with $\mathrm{hSBA}$ titers $\geq$ LLOQ to different numbers of primary MenB test strains before vaccination, 1 month after 2 doses (0,2 months), and 1 month after 3 doses (0, 2, 6 months). LLOQ was defined as 1:8 for the test strain expressing FHbp variant A22 and 1:16 for the test strains expressing FHbp variants A56, B24, and B44. The

clinical efficacy study of a meningococcal vaccine. As a result, clinical studies of MenB vaccines measure immune responses by hSBA, which is the accepted surrogate of vaccine efficacy and has been used to license a variety of meningococcal vaccines [17, 32, 33]. MenBFHbp contains 2 recombinant lipidated protein FHbp antigens [13]; this protein has extensive sequence diversity, with gene sequences segregating into 2 subfamilies (A and B) [23, 34, 35]. Serologic evidence of broad coverage of FHbp variants by MenB vaccines targeting this protein is therefore essential to ensure adequate clinical efficacy against circulating disease-causing MenB strains.

Although there is no universally accepted standard of broad protection against MenB disease, 2 phase 3 studies of MenB-FHbp established a rigorous framework to demonstrate the vaccine's breadth of coverage [29]. Immunogenicity endpoints measuring protective responses against 4 primary and 10 additional MenB test strains in these studies used a threshold of protection that was more stringent than the accepted correlate of protection

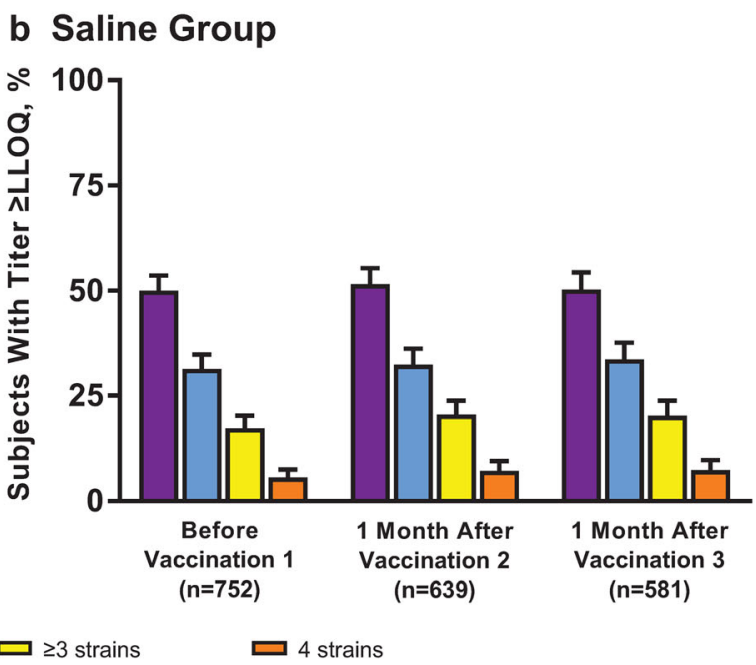

number of subjects included at each time point is indicated below the $x$-axis. FHbp factor $\mathrm{H}$ binding protein, $b S B A$ serum bactericidal antibody assay using human complement, LLOQ lower limit of quantitation, MenB Neisseria meningitidis serogroup B, MenB-FHbp bivalent rLP2086, Trumenba ${ }^{\circledR}$. Error bars represent the $95 \%$ CIs calculated using the Clopper-Pearson method

(i.e., LLOQ determined as titers $1: 8$ or $1: 16$ rather than $1: 4$ ), along with a composite endpoint that reflected responses to all 4 primary test strains combined [17, 18, 29]; these endpoints may therefore actually underestimate breadth of coverage. The high threshold defined for a protective immune response and the evaluation of the composite response combined with the diversity of the MenB test strain panel enabled an estimate of broad coverage elicited by MenB-FHbp. The rigor of this methodology is particularly important in that the method used to evaluate breadth of coverage of MenB vaccines may influence the ability to predict real-life clinical effectiveness. Coverage estimates for another licensed MenB vaccine, MenB-4C (Bexsero ${ }^{\circledR}, 4$ CMenB; GlaxoSmithKline Vaccines, Srl, Siena, Italy), primarily used the Meningococcal Antigen Typing System and a genotyping test to determine immunogenicity against the vaccine antigens (i.e., FHbp, neisserial adhesin A, neisserial heparin-binding antigen, and outer membrane vesicles) [14, 36, 37]. However, a recent analysis of pre- and postvaccination responses conducted during a U.S. 
Table 1 Subjects with hSBA titers $<$ LLOQ at 1 month post-dose 3 to all primary strains and to additional test strains assessed in certain individuals

\begin{tabular}{llll}
\hline Subject identifier $^{\mathbf{a}}$ & Sex & Continent & Strains evaluated for which hSBA LLOQ $^{\mathbf{b}}$ \\
\hline Adolescents & & & \\
A & Male & Europe & A22, A56, B24, B44 \\
B & Female & North America & A22, A56, B24, B44, A15, A19, A29 \\
C & Female & North America & A22, A56, B24, B44, B03, B09, B15, B16 \\
Young adults & & & \\
D & Female & North America & A22, A56, B24, B44 \\
E & Female & Europe & A22, A56, B24, B44 \\
F & Female & Europe & A22, A56, B24, B44 \\
G & Male & North America & A22, A56, B24, B44 \\
H & Female & North America & A22, A56, B24, B44 \\
I & Female & North America & A22, A56, B24, B44, A15, A19, A29 \\
\hline
\end{tabular}

Bold text indicates individuals in whom primary and additional test strains were assessed

$F H b p$ factor $\mathrm{H}$ binding protein, $h S B A$ serum bactericidal antibody assay using human complement, $L L O Q$ lower limit of quantitation

a Subject identifier for the current analysis

${ }^{b}$ Strains are indicated by FHbp variant

${ }^{c}$ hSBA titer $=4$ at 1 month post-dose 3

university vaccination campaign with MenB-4C in response to an outbreak found the seroconversion (i.e., $\geq 4$-fold increase in titers) rate in hSBA for the particular outbreak strain, which closely matched 2 of the MenB-4C antigens, to be only $20.3 \%$ following completion of the licensed 2-dose schedule $[14,38]$; this was approximately 4-5 times lower than that for 2 MenB-4C antigen-specific indicator strains (83.0-98.3\%). These findings reiterate the requirement for a rigorous approach to demonstrate breadth of coverage of MenB vaccines, and that using vaccine-heterologous, diverse test strain panels is essential.

By comparison with the MenB-4C findings, results from the phase 3 MenB-FHbp studies indicated that although $\mathrm{FHbp}$ is naturally diverse, a bivalent vaccine containing FHbp from each immunologically distinct subfamily (subfamilies A and B) is sufficient for broad coverage against vaccine-heterologous strains, with large percentages of adolescents and young adults $(78.8-90.2 \%$ and $78.9-89.7 \%$, respectively) exhibiting $\geq 4$-fold hSBA titer increases against each of the 4 primary strains after vaccination [29]. Broad coverage against diverse MenB test strains elicited by the vaccine was further supported by the large percentages $(82.7-84.5 \%)$ of adolescents and young adults who had hSBA titers $\geq$ LLOQ for all 4 primary test strains combined at 1 month after dose 3 . Further, an exploratory analysis in a subset of subjects from previous MenB-FHbp studies also examined hSBA responses against MenB strains from the same university outbreak as described above, reporting $44-67 \%$ and $78-100 \%$ of adolescents had $\geq 4$-fold hSBA titer increases after 2 and 3 doses of MenB-FHbp, respectively; no bactericidal activity was detected pre-vaccination (unpublished data). The current post hoc analysis aimed to further expand this evidence of MenB-FHbp-mediated broad protection by evaluating protective responses to more 
extensive arrays of pathogenic strains in the test strain panel.

In the current analysis, subjects were tested against a total of either 7 ( 2 subsets) or 8 (1 subset) primary plus additional strains. At 1 month post-dose $3,70.5-85.8 \%$ of adolescents and $67.5-81.4 \%$ of young adults had responses to at least 7 strains combined. Furthermore, a high percentage of subjects without hSBA titers $\geq$ LLOQ to all 4 primary test strains or at least 7 strains combined still achieved protective responses to multiple test strains (e.g., $92.8 \%$ of adolescents and $94.0 \%$ of young adults responded to at least 3 primary test strains combined at 1 month post-dose 3; 93.7-95.7\% of adolescents and 91.7-95.0\% of young adults responded to at least 5 strains combined at 1 month post-dose 3 ). This indicates that subjects not responding to 1 or more of the primary test strains would likely still benefit from vaccination because of demonstrated coverage of additional strains. Consistent with this, only 3 subjects out of all vaccinees did not exhibit vaccine-elicited immune responses against any of the strains tested. For all analyses, percentages of subjects responding in the MenB-FHbp groups substantially increased from baseline to 1 month postdose 2 , and further increased 1 month post-dose 3 ; percentages in the control groups, where only the primary strains were examined, remained similar to baseline. As expected, when data were analyzed using the accepted correlate of protection (hSBA titer $\geq 1: 4$ ), slightly higher response rates were observed. These results collectively reiterate the capacity for MenB-FHbp to provide broad protective coverage against diverse MenB disease isolates.

Higher percentages of young adults had prevaccination hSBA titers $\geq$ LLOQ to the primary and additional test strains compared with adolescents, which is consistent with observations suggesting that exposure to meningococcus increases over an individual's life [18]. Importantly, percentages were low for both age groups: $1.4 \%$ of adolescents and $6.7 \%$ of young adults had pre-vaccination hSBA titers $\geq$ LLOQ for all 4 primary test strains. In addition, very few adolescents $(0.4-1.7 \%)$ and young adults $(0.8-9.1 \%)$ who were tested against 7 or
8 strains combined had pre-vaccination hSBA titers $\geq$ LLOQ against at least 7 test strains. These observations confirm that, with limited exceptions, a broad hSBA response to MenB is only achieved following MenB-FHbp vaccination [39].

It has been hypothesized that use of an FHbp-based vaccine could potentially lead to increased prevalence of escape mutants that evade bactericidal activity by lacking FHbp or expressing it at insufficient levels [40]. However, FHbp is an important virulence factor for meningococci, as highlighted by the observation that $\leq 1 \%$ of invasive MenB isolates lacked the $\mathrm{fHbp}$ gene and that these isolates all belonged to clonal complexes that were rarely associated with MenB disease [41]. In addition, an intact $f H b p$ gene was detected in $100 \%$ of 650 sequenced isolates from the US Centers for Disease Control and Prevention's MenB strain collection [42]. Further, another study found that $91.4 \%$ of strains expressed FHbp at levels consistently predicted to be killed in hSBA by sera from subjects vaccinated with MenB-FHbp; strains with lower expression levels were also susceptible [19]. These findings are underscored by a study demonstrating that both healthy individuals with meningococcal carriage and patients with invasive disease can robustly mount antibodies to FHbp despite harboring meningococcal strains with in vitro FHbp expression levels below the cutoff [43]. The evidence supporting the potential of vaccine escape mutants following widespread use of MenB-FHbp is not available at present, but the current understanding of the role of FHbp in the virulence of serogroup $\mathrm{B}$ meningococci suggests that this is unlikely.

National vaccine technical committees face the key challenge of assessing data on the extent of protective immune coverage afforded by MenB vaccines against IMD-causing strains circulating in their regions. Approaches that rely on using pooled immune sera from a small number of vaccinees tested against a limited number of meningococcal antigen-specific strains do not provide an informative measure of population immune response [44]. In contrast, the dataset described in the current analysis evaluated individual immune sera using 
hSBA assays against a panel of 14 globally diverse MenB strains in 2 large randomized control clinical trials [29]. In addition, the post hoc analyses demonstrate that a large percentage of the individual vaccinees responded to as many as 8 strains combined from the test panel. Taken together, these data should engender confidence among immunization policymakers in the ability of MenB-FHbp vaccine to confer broad protection against MenB disease.

The findings of this analysis should be considered along with the potential limitations of the primary studies [29], including that vaccine efficacy assessments were precluded by low MenB disease incidence, and that the persistence of antibody responses was not evaluated. However, as previously noted, short-term antibody responses are the accepted surrogate of efficacy for meningococcal vaccines, while data on the persistence of immune responses for up to 4 years following primary vaccination and responses to a booster dose in adolescents are already published [45]. In addition, this study is limited by the post hoc nature of the analysis; although exploratory, our findings reinforce the original study conclusions that MenB-FHbp provides broad coverage against MenB strains expressing diverse FHbp variants [29].

\section{CONCLUSIONS}

The findings of this post hoc analysis extend those previously reported [29] and further support the capacity for MenB-FHbp to provide broad protective coverage against diverse MenB strains. Accordingly, these results provide additional assurance that MenB-FHbp is protective against contemporary disease-causing MenB strains.

\section{ACKNOWLEDGEMENTS}

We thank all study participants for taking part in the clinical studies that provided data for this analysis.

Funding. This work was funded by Pfizer Inc. Pfizer Inc also funded Rapid Service
Fees. All authors had full access to all of the data in this study and take complete responsibility for the integrity of the data and accuracy of the data analysis.

Medical Writing, Editorial, and Other Assistance. These studies were sponsored by Pfizer Inc. The authors thank Laura J. York for providing helpful feedback on the manuscript. Medical writing support was provided by Emily Stackpole, PhD, and Judith Kandel, PhD, of ICON plc (North Wales, PA, USA) and was funded by Pfizer Inc.

Authorship. All named authors meet the International Committee of Medical Journal Editors (ICMJE) criteria for authorship for this article, take responsibility for the integrity of the work as a whole, and have given their approval for this version to be published.

Prior Presentation. This work was presented previously at the 2018 International Pathogenic Neisseria Conference, September 23-28, 2018; Monterey, CA, USA (poster) and at the 2019 Pediatric Academic Societies meeting, April 24May 1, 2019; Baltimore, MD, USA (poster).

Disclosures. Johannes Beeslaar, Judith Absalon, Annaliesa S. Anderson, Joseph J. Eiden, Paul Balmer, Shannon L. Harris, Thomas R. Jones, Robert E. O'Neill, Jean-Louis Pregaldien, David Radley, Roger Maansson, John Ginis, Amit Srivastava, and John L. Perez are current or former employees of Pfizer Inc and may hold stock or stock options. At the time of study, Joseph J. Eiden, Shannon L. Harris, and Thomas R. Jones were employees of Pfizer Inc. Shannon L. Harris is currently an employee of Seqirus (Cambridge, MA, USA). Joseph J. Eiden and Thomas R. Jones are currently retired from Pfizer Inc.

Compliance with Ethics Guidelines. Protocols for both previously conducted studies used for analysis here were approved by the institutional review boards and/or independent ethics committees for the respective investigational centers that participated. Written informed consent was obtained from all subjects or their legal guardian prior to enrollment and any 
study-related procedures. The present analysis is based on existing data collected from these studies and therefore did not involve any new data collection or new studies with human subjects performed by any of the authors.

Data Availability. Upon request, and subject to certain criteria, conditions and exceptions (see https://www.pfizer.com/science/ clinical-trials/trial-data-and-results for more information), Pfizer will provide access to individual de-identified participant data from Pfizersponsored global interventional clinical studies conducted for medicines, vaccines and medical devices (1) for indications that have been approved in the US and/or EU or (2) in programs that have been terminated (i.e., development for all indications has been discontinued). Pfizer will also consider requests for the protocol, data dictionary, and statistical analysis plan. Data may be requested from Pfizer trials 24 months after study completion. The deidentified participant data will be made available to researchers whose proposals meet the research criteria and other conditions, and for which an exception does not apply, via a secure portal. To gain access, data requestors must enter into a data access agreement with Pfizer.

Open Access. This article is licensed under a Creative Commons Attribution-NonCommercial 4.0 International License, which permits any non-commercial use, sharing, adaptation, distribution and reproduction in any medium or format, as long as you give appropriate credit to the original author(s) and the source, provide a link to the Creative Commons licence, and indicate if changes were made. The images or other third party material in this article are included in the article's Creative Commons licence, unless indicated otherwise in a credit line to the material. If material is not included in the article's Creative Commons licence and your intended use is not permitted by statutory regulation or exceeds the permitted use, you will need to obtain permission directly from the copyright holder. To view a copy of this licence, visit http://creativecommons.org/licenses/by$\mathrm{nc} / 4.0 /$.

\section{REFERENCES}

1. Cohn AC, MacNeil JR, Clark TA, et al. Prevention and control of meningococcal disease: recommendations of the Advisory Committee on Immunization Practices (ACIP). MMWR Recomm Rep. Available at: https://www.cdc.gov/mmwr/preview/ mmwrhtml/rr6202a1.htm. Accessed 3 Feb 2020.

2. Centers for Disease Control and Prevention. Enhanced Meningococcal Disease Surveillance Report, 2018. Available at: https://www.cdc.gov/ meningococcal/downloads/NCIRD-EMS-Report2018.pdf. Accessed 7 Jan 2020.

3. European Centre for Disease Prevention and Control. Surveillance Atlas of Infectious Diseases. Available at: https://ecdc.europa.eu/en/surveillanceatlas-infectious-diseases. Accessed 14 Apr 2020.

4. Pace D, Pollard AJ. Meningococcal disease: clinical presentation and sequelae. Vaccine. 2012;30:B3-9.

5. Thompson MJ, Ninis N, Perera R, et al. Clinical recognition of meningococcal disease in children and adolescents. Lancet. 2006;367:397-403.

6. Sadarangani M, Scheifele DW, Halperin SA, et al. Outcomes of invasive meningococcal disease in adults and children in Canada between 2002 and 2011: a prospective cohort study. Clin Infect Dis. 2015;60:e27-35.

7. Borrow R, Alarcon P, Carlos J, et al. The Global Meningococcal Initiative: global epidemiology, the impact of vaccines on meningococcal disease and the importance of herd protection. Expert Rev Vaccines. 2017;16:313-28.

8. Sridhar S, Greenwood B, Head C, et al. Global incidence of serogroup $\mathrm{B}$ invasive meningococcal disease: a systematic review. Lancet Infect Dis. 2015;15:1334-46.

9. European Centre for Disease Prevention and Control. Annual Epidemiological Report 2016 - Invasive meningococcal disease. http://ecdc.europa. $\mathrm{eu} / \mathrm{en} /$ healthtopics/meningococcal/Pages/Annualepidemiological-report-2016.aspx. Accessed $24 \mathrm{Apr}$ 2017.

10. Menveo ${ }^{\circledR}$ (meningococcal [groups A, C, Y and W-135] oligosaccharide diphtheria $\mathrm{CRM}_{197}$ conjugate vaccine). Full Prescribing Information, Novartis, Sovicille, Italy, 2013.

11. Menactra ${ }^{\circledR}$ (Meningococcal [Groups A, C, Y and W-135] Polysaccharide Diphtheria Toxoid Conjugate Vaccine). Full Prescribing Information, Sanofi Pasteur Inc., Swiftwater, PA, 2018. 
12. Pfizer Canada Inc. Nimenrix Product Monograph. Kirkland, QC: Pfizer Canada Inc; 2016.

13. Trumenba ${ }^{\circledR}$ (meningococcal group B vaccine). Full Prescribing Information, Wyeth Pharmaceuticals Inc (a subsidiary of Pfizer Inc), Philadelphia, PA, 2018.

14. Bexsero ${ }^{\circledR}$ (Meningococcal Group B Vaccine). Full Prescribing Information, GSK Vaccines, Srl, Siena, Italy, 2018.

15. Bruge J, Bouveret-Le Cam N, Danve B, Rougon G, Schulz D. Clinical evaluation of a group B meningococcal N-propionylated polysaccharide conjugate vaccine in adult, male volunteers. Vaccine. 2004;22:1087-96.

16. Wyle FA, Artenstein MS, Brandt BL, et al. Immunologic response of man to group $B$ meningococcal polysaccharide vaccines. J Infect Dis. $1972 ; 126: 514-21$.

17. Borrow R, Balmer P, Miller E. Meningococcal surrogates of protection-serum bactericidal antibody activity. Vaccine. 2005;23:2222-7.

18. Goldschneider I, Gotschlich EC, Artenstein MS. Human immunity to the meningococcus. I. The role of humoral antibodies. J Exp Med. 1969;129: 1307-26.

19. McNeil LK, Donald RGK, Gribenko A, et al. Predicting the susceptibility of meningococcal serogroup B isolates to bactericidal antibodies elicited by bivalent rLP2086, a novel prophylactic vaccine. MBio. 2018;9:e00036-18.

20. Lucidarme J, Comanducci $\mathrm{M}$, Findlow J, et al. Characterization of fHbp, nhba (gna2132), nadA, porA, and sequence type in group B meningococcal case isolates collected in England and Wales during January 2008 and potential coverage of an investigational group B meningococcal vaccine. Clin Vaccine Immunol. 2010;17:919-29.

21. Toneatto D, Ismaili S, Ypma E, et al. The first use of an investigational multicomponent meningococcal serogroup B vaccine (4CMenB) in humans. Hum Vaccin. 2011;7:646-53.

22. McNeil LK, Zagursky R, Shuo L, et al. The role of factor $\mathrm{H}$ binding protein in Neisseria meningitidis virulence and its potential as a vaccine candidate to broadly protect against meningococcal disease. Microbiol Mol Biol Rev. 2013;77:234-52.

23. Neisseria Factor $\mathrm{H}$ binding protein sequence typing. Available at: https://pubmlst.org/neisseria/fHbp/. Accessed 11 Oct 2018.
24. US Food and Drug Administration. Summary Basis for Regulatory Action. Available at: https://way back.archive-it.org/7993/20190425012126/https:// www.fda.gov/downloads/BiologicsBloodVaccines/ Vaccines/ApprovedProducts/UCM424125.pdf. Accessed 8 Jul 2020.

25. European Medicines Agency. EPAR Summary for the Public: Trumenba. http://www.ema.europa.eu/ docs/en_GB/document_library/EPAR_-_Summary_ for_the_public/human/004051/WC500228998.pdf. Accessed 12 July 2019.

26. Pfizer Canada Inc. Product monograph: Trumenba ${ }^{\mathrm{TM}}$. Pfizer Canada Inc. https://pdf.hres.ca/dpd_pm/ 00041515.PDF. Accessed 11 Oct 2018.

27. Australian Government Department of Health Therapeutic Goods Administration. Prescription Medicines: Registration of New Chemical Entities in Australia. https://www.tga.gov.au/prescriptionmedicines-registration-new-chemical-entities-australia. Accessed 11 Oct 2018.

28. Trumenba (MenB-FHbp; bivalent rLP2086). Full Prescribing Information, Pfizer Chile S.A., Santiago, Chile, 2016.

29. Ostergaard L, Vesikari T, Absalon J, et al. A bivalent meningococcal B vaccine in adolescents and young adults. N Engl J Med. 2017;377:2349-62.

30. Zlotnick GW, Jones TR, Liberator P, et al. The discovery and development of a novel vaccine to protect against Neisseria meningitidis serogroup B disease. Hum Vaccine Immunother. 2015;11:5-13.

31. Borrow R, Carlone GM, Rosenstein $\mathrm{N}$, et al. Neisseria meningitidis group B correlates of protection and assay standardization-international meeting report Emory University, Atlanta, Georgia, United States, 16-17 March 2005. Vaccine. 2006;24:5093-107.

32. Frasch CE, Borrow R, Donnelly J. Bactericidal antibody is the immunologic surrogate of protection against meningococcal disease. Vaccine. 2009;27: B112-6.

33. Tan LK, Carlone GM, Borrow R. Advances in the development of vaccines against Neisseria meningitidis. N Engl J Med. 2010;362:1511-20.

34. Fletcher LD, Bernfield L, Barniak V, et al. Vaccine potential of the Neisseria meningitidis 2086 lipoprotein. Infect Immun. 2004;72:2088-100.

35. Murphy E, Andrew L, Lee KL, et al. Sequence diversity of the factor $\mathrm{H}$ binding protein vaccine candidate in epidemiologically relevant strains of serogroup B Neisseria meningitidis. J Infect Dis. 2009;200:379-89. 
36. Donnelly J, Medini D, Boccadifuoco G, et al. Qualitative and quantitative assessment of meningococcal antigens to evaluate the potential strain coverage of protein-based vaccines. Proc Natl Acad Sci USA. 2010;107:19490-5.

37. Medini D, Stella M, Wassil J. MATS: global coverage estimates for 4CMenB, a novel multicomponent meningococcal B vaccine. Vaccine. 2015;33:2629-36.

38. Basta N, Wolfson J, Mahmoud A, et al. 4CMenB vaccine immunogenicity up to 1 year after vaccination among university students. Presented at: International Pathogenic Neisseria Conference, September 23-28, 2018; Asilomar, CA, USA.

39. Lucidarme J, Lekshmi A, Parikh SR, et al. Frequent capsule switching in 'ultra-virulent' meningococci-are we ready for a serogroup B ST-11 complex outbreak? J Infect. 2017;75:95-103.

40. Lewis LA, Ngampasutadol J, Wallace R, et al. The meningococcal vaccine candidate neisserial surface protein A (NspA) binds to factor $\mathrm{H}$ and enhances meningococcal resistance to complement. PLoS Pathog. 2010;6:e1001027.
41. Lucidarme J, Tan L, Exley RM, et al. Characterization of Neisseria meningitidis isolates that do not express the virulence factor and vaccine antigen factor $\mathrm{H}$ binding protein. Clin Vaccine Immunol. 2011;18:1002-14.

42. Wang X, Cohn A, Comanducci M, et al. Prevalence and genetic diversity of candidate vaccine antigens among invasive Neisseria meningitidis isolates in the United States. Vaccine. 2011;29:4739-44.

43. Ala'Aldeen DA, Flint M, Oldfield NJ, et al. Human antibody responses to the meningococcal factor $\mathrm{H}$ binding protein (LP2086) during invasive disease, colonization and carriage. Vaccine. 2010;28: 7667-75.

44. Donald RG, Hawkins JC, Hao L, et al. Meningococcal serogroup B vaccines: estimating breadth of coverage. Hum Vaccine Immunother. 2017;13: 255-65.

45. Vesikari T, Ostergaard L, Beeslaar J, et al. Persistence and 4-year boosting of the bactericidal response elicited by two- and three-dose schedules of MenBFHbp: a phase 3 extension study in adolescents. Vaccine. 2019;37:1710-1719. 